\title{
Recent nucleosynthesis results from INTEGRAL
}

\author{
G. Weidenspointner \\ Centre d'Etude Spatiale des Rayonnements, 9, avenue du Colonel-Roche, BP 4346, 31028 \\ Toulouse Cedex 4, France \\ email: Georg.Weidenspointner@cesr.fr
}

\begin{abstract}
Since its launch in October 2002, ESA's INTEGRAL observatory has enabled significant advances to be made in the study of Galactic nucleosynthesis. In particular, the imaging Ge spectrometer SPI combines for the first time the diagnostic powers of high resolution gamma-ray line spectroscopy and moderate spatial resolution. This review summarizes the major nucleosynthesis results obtained with INTEGRAL so far. Positron annihilation in our Galaxy is being studied in unprecented detail. SPI observations yield the first sky maps in both the $511 \mathrm{keV}$ annihilation line and the positronium continuum emission, and the most accurate spectrum at $511 \mathrm{keV}$ to date, thereby imposing new constraints on the source(s) of Galactic positrons which still remain(s) unidentified. For the first time, the imprint of Galactic rotation on the centroid and shape of the $1809 \mathrm{keV}$ gamma-ray line due to the decay of ${ }^{26} \mathrm{Al}$ has been seen, confirming the Galactic origin of this emission. SPI also provided the most accurate determination of the gamma-ray line flux due to the decay of ${ }^{60} \mathrm{Fe}$. The combined results for ${ }^{26} \mathrm{Al}$ and ${ }^{60} \mathrm{Fe}$ have important implications for nucleosynthesis in massive stars, in particular Wolf-Rayet stars. Both IBIS and SPI are searching the Galactic plane for young supernova remnants emitting the gamma-ray lines associated with radioactive ${ }^{44} \mathrm{Ti}$. None have been found so far, which raises important questions concerning the production of ${ }^{44} \mathrm{Ti}$ in supernovae, the Galactic supernova rate, and the Galaxy's chemical evolution.
\end{abstract}

Keywords. gamma rays: observations; Galaxy: general; nuclear reactions, nucleosynthesis, abundances

\section{Introduction}

Gamma-ray line astronomy has opened a new and unique window for studying nucleosynthesis in our Galaxy. The singular advantage of gamma-ray spectroscopy over other observations is that it offers the opportunity to detect directly and identify uniquely individual isotopes at their birthplaces. The rate at which radioactive decays proceed is in general unaffected by the physical conditions in their environment, such as temperature or density. The interstellar medium is not dense enough to attenuate gamma rays, so that radioactive decays can be observed throughout our Galaxy. Recent reviews on implications of gamma-ray observations for nucleosynthesis in our Galaxy can be found in Diehl, Prantzos, \& von Ballmoos (2005) and Prantzos (2005).

\section{Results}

The nucleosynthesis results presented in the following have all been obtained from observations with the two main instruments on board the INTEGRAL observatory: the spectrometer SPI and the imager IBIS (for details regarding the instruments, see Kretschmar (2005) and references therein). These two instruments are complementary in their characteristics, providing an unprecedented view of the Universe at hard X-ray 

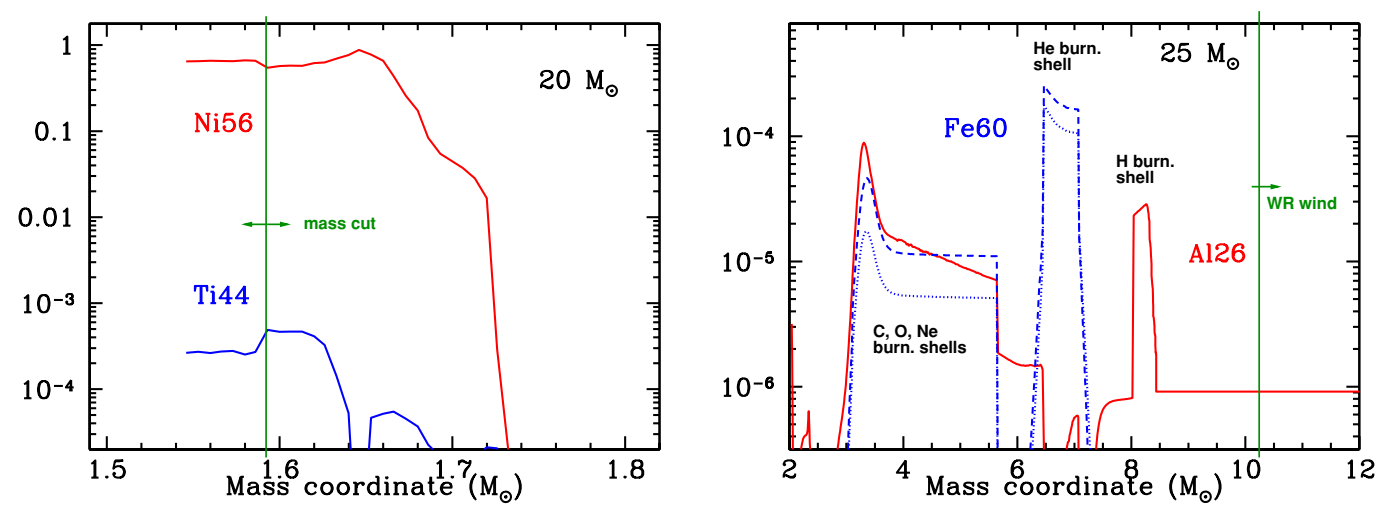

Figure 1. Left panel: radial abundance profiles (mass fractions) of ${ }^{56} \mathrm{Ni}$ and ${ }^{44} \mathrm{Ti}$ inside a $20 \mathrm{M}_{\odot}$ star after the passage of the shock front. Right panel: radial abundance profiles (mass fractions) of ${ }^{26} \mathrm{Al}$ and ${ }^{60} \mathrm{Fe}$ inside a $25 \mathrm{M}_{\odot}$ star after the passage of the shock front. Both figures were adapted from Prantzos (2005).

and soft gamma-ray energies. The imaging Ge spectrometer SPI offers high spectral resolution of about $2.1 \mathrm{keV}$ FWHM at $511 \mathrm{keV}$ combined for the first time with moderate spatial resolution (FWHM about $3^{\circ}$ ). The imager IBIS offers excellent spatial resolution of about $12^{\prime}$ FWHM at moderate spectral resolution (FWHM about $38 \mathrm{keV}$ at $511 \mathrm{keV}$ ).

$$
\text { 2.1. }{ }^{44} \mathrm{Ti}
$$

\subsection{1. ${ }^{44} \mathrm{Ti}$ production sites and processes}

The radioisotope ${ }^{44} \mathrm{Ti}$ is primarily produced in the so-called $\alpha$-rich freeze-out of material initially in nuclear statistical equilibrium. The main site for $\alpha$-rich freeze-out to occur is thought to be the innermost layers of core-collapse supernovae (ccSNe), although sub-Chandrasekhar mass white dwarf Type Ia SNe have also been proposed (Woosley \& Weaver 1994).

The ${ }^{44} \mathrm{Ti}$ yield of ccSNe is notoriously difficult to calculate because it depends sensitively on the so-called mass cut, the explosion energy, and the (a)symmetry of the explosion. The mass cut, which has not yet been successfully calculated and is illustrated in the left panel of Fig. 1, is the notional surface separating material that is ejected from material that will fall back onto the compact remnant (neutron star or black hole) of the explosion. ${ }^{44} \mathrm{Ti}$ is believed to be produced in the deepest layers of the exploding star that may be ejected, depending on the precise location of the mass cut. The amount of synthesized ${ }^{44} \mathrm{Ti}$ also depends sensitively on the explosion energy and (a)symmetry. Theoretical calculations indicate that both increased explosion energy and increased asymmetry result in an increased ${ }^{44} \mathrm{Ti}$ yield.

Observationally, the presence of the radioisotope ${ }^{44} \mathrm{Ti}$ is revealed to the gamma-ray astronomer through the emission of three gamma-ray lines. The decay ${ }^{44} \mathrm{Ti} \rightarrow{ }^{44} \mathrm{Sc}$ $\left(\tau_{44} \mathrm{Ti} \sim 90 \mathrm{y}\right)$ gives rise to gamma rays at $67.9 \mathrm{keV}$ and $78.4 \mathrm{keV}$; the subsequent decay ${ }^{44} \mathrm{Sc} \rightarrow{ }^{44} \mathrm{Ca}\left(\tau_{44} S c \sim 5.7 \mathrm{~h}\right)$ gives rise to a line at $1157.0 \mathrm{keV}$.

The astrophysical interest in ${ }^{44} \mathrm{Ti}$ is two-fold. Clearly, the amount and the velocity of ${ }^{44} \mathrm{Ti}$ is a very powerful probe of the explosion mechanism and dynamics of ccSNe, which are still poorly understood. In addition, the ${ }^{44} \mathrm{Ti}$ gamma-ray line emission is an ideal indicator of young SN remnants (SNRs). The lifetime is about $90 \mathrm{y}$, which roughly coincides with the expected recurrence time interval for ccSNe in our Galaxy. It is therefore 


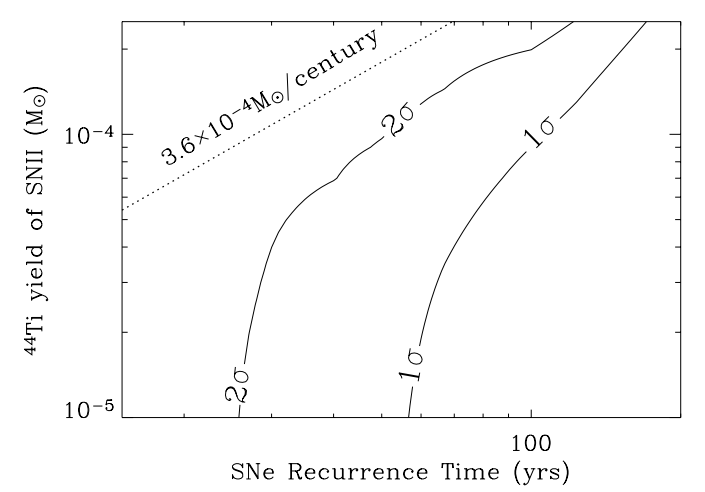

Figure 2. Contours denoting the probability that IBIS/ISGRI would have detected at least one ${ }^{44} \mathrm{Ti}$ point source as a function of the ${ }^{44} \mathrm{Ti}$ yield of Type II SNe and the Type II SN recurrence time (figure adapted from Renaud et al. 2004). The dotted line represents the present-day ${ }^{44} \mathrm{Ti}$ production rate that is required to explain the solar system abundance of ${ }^{44} \mathrm{Ca}$, assuming that is solely produced as ${ }^{44} \mathrm{Ti}$ in Type II SNe.

expected that with a sufficiently sensitive instrument a few young SNRs should be visible in our Galaxy at the current epoch.

\subsubsection{Search for young SNRs in the inner Galaxy}

The most sensitive search to date for young SNRs at gamma-ray energies was performed by Renaud et al. (2004) who used the first year of INTEGRAL observations to search for $68 \mathrm{keV}$ and $78 \mathrm{keV}$ line emission in the inner Galaxy with the imager IBIS. This search addresses a long-standing puzzle linking the Galactic SN rate and Galactic chemical evolution: given current estimates of the present-day rates of thermonuclear and ccSNe and their yields, these events can only account for about $1 / 3$ of the solar ${ }^{44} \mathrm{Ca}$ abundance based on chemical evolution models and assuming that all ${ }^{44} \mathrm{Ca}$ is formed as ${ }^{44} \mathrm{Ti}$ (Leising \& Share 1994). At the same time, given these SN properties, combined with models for their Galactic distribution, past missions should have detected a few young SNRs even with their lower sensitivities - and detections were certainly expected for the unprecedented limiting point source sensitivity achieved with IBIS. However, as was the case in less sensitive previous searches, none have been found.

To assess the implications of the non-detection of young SNRs, Renaud et al. (2004) estimated the probability that at least one ${ }^{44} \mathrm{Ti}$ point source is detectable by generating Monte Carlo distributions using current estimates of the rates of thermonuclear and ccSNe, of their yields, and of their Galactic distribution; SN explosions were simulated as a function of the recurrence time and the ${ }^{44} \mathrm{Ti}$ yield of Type II SNe. A typical result is depicted in Fig. 2.

The Monte Carlo study rules out two obvious solutions to the ${ }^{44} \mathrm{Ca}$ puzzle. The estimated Galactic ${ }^{44} \mathrm{Ti}$ production rate of ccSNe could in principle be increased by postulating that the Galactic SN rate has been underestimated, or by postulating that the ${ }^{44} \mathrm{Ti}$ yields have been underestimated. Neither solution is viable, because in order to account for the solar ${ }^{44} \mathrm{Ca}$ abundance by $\mathrm{SNe}$ a present-day production rate of $3.6 \times 10^{-4} \mathrm{M}_{\odot}$ century $^{-1}$ is required - which is excluded at a greater than $95 \%$ confidence level for any acceptable combination of SN rates and ${ }^{44} \mathrm{Ti}$ yields. A third solution, compatible with existing data, is the existence of another, rare but high yield, Galactic ${ }^{44} \mathrm{Ti}$ source such as sub-Chandrasekhar mass white dwarf Type Ia SNe. The latest search for young SNRs with IBIS therefore highlights important open questions concerning the chemical evolution of our Galaxy, the production of ${ }^{44} \mathrm{Ti}$ in SNe, and the Galactic SN rate (Renaud et al. 2004). 


\section{2. ${ }^{26} \mathrm{Al}$ and ${ }^{60} \mathrm{Fe}$}

\subsection{1. ${ }^{26} \mathrm{Al}$ and ${ }^{60} \mathrm{Fe}$ production sites and processes}

${ }^{26} \mathrm{Al}$ and ${ }^{60} \mathrm{Fe}$ are produced hydrostatically as well as explosively in massive stars. The production of ${ }^{26} \mathrm{Al}$ requires free protons, hence it is mainly produced in the $\mathrm{H}, \mathrm{Ne}$, and $\mathrm{C}$ burning layers; the production of ${ }^{60} \mathrm{Fe}$ requires free neutrons, hence it is mainly produced in $\mathrm{C}$, Ne, and He burning layers, as indicated in the right panel of Fig. 1. Products of hydrostatic nucleosynthesis can escape the massive star through stellar winds, in particular during the Wolf-Rayet (WR) phase of the most massive stars. The ${ }^{26} \mathrm{Al}$, being produced in $\mathrm{H}$ burning layers near the surface, can escape most easily. When the star explodes in a ccSN, about equal amounts of ${ }^{26} \mathrm{Al}$ and ${ }^{60} \mathrm{Fe}$ are ejected. The yields of ${ }^{60} \mathrm{Fe}$ and in particular of ${ }^{26} \mathrm{Al}$ are hard to predict because of the important role of stellar winds, which in turn depend e.g. on the metallicity of the star or its rotation.

Both radioisotopes can be observed by gamma-ray line spectroscopy. The decay of ${ }^{26} \mathrm{Al}\left(\tau_{26} \mathrm{Al} \sim 1.1 \times 10^{6} \mathrm{y}\right)$ gives rise to a single gamma-ray line at $1808.6 \mathrm{keV}$. The decay ${ }^{60} \mathrm{Fe} \rightarrow{ }^{60} \mathrm{Co}\left(\tau_{60} \mathrm{Fe} \sim 2.2 \times 10^{6} \mathrm{y}\right)$ is revealed by a line at $58.6 \mathrm{keV}$, the subsequent decay ${ }^{60} \mathrm{Co} \rightarrow{ }^{60} \mathrm{Ni}\left(\tau^{60} \mathrm{Co}_{o} \sim 7.6 \mathrm{y}\right)$ is revealed by two lines at $1173.2 \mathrm{keV}$ and $1332.5 \mathrm{keV}$.

Astrophysically, the subtle differences in the production zones of the radioisotopes ${ }^{26} \mathrm{Al}$ and ${ }^{60}$ Fe render their combined study an important probe of massive star nucleosynthesis. Furthermore, since the lifetimes of these two radioisotopes are much shorter than Galactic evolution timescales but are large enough to accumulate in the ISM, ${ }^{26} \mathrm{Al}$ and ${ }^{60} \mathrm{Fe}$ are ideally suited to study the sites and the distribution of recent Galactic star formation.

\subsection{2. ${ }^{26}$ Al in the inner Galaxy}

The sky distribution of Galactic ${ }^{26} \mathrm{Al}$ was first mapped using the COMPTEL instrument; it was found to be very similar to that of massive stars in our Galaxy, establishing the massive star origin of ${ }^{26} \mathrm{Al}$ (Knödlseder et al. 1999). However, the relative importance of WR star winds and of ccSNe for seeding the ISM remained unclear. Early high-resolution spectroscopy with the balloon borne GRIS instrument by Naya et al. (1996) yielded an unexpectedly large line width of about $5.4 \mathrm{keV}$ FWHM and complicated rather than clarified the origin of ${ }^{26} \mathrm{Al}$ as such substantial line broadening could only be explained by rather unusual circumstances (Sturner \& Naya 1999). Spatially resolved spectroscopy, as afforded by the imaging spectrometer SPI, is expected to resolve the issue, as it will make it possible to probe the imprint of the dynamics of the ISM local to the ${ }^{26} \mathrm{Al}$ sources and that of Galactic differential rotation on shape and centroid of the $1808.6 \mathrm{keV}$ line (modelled e.g. by Kretschmer et al. 2003).

Using the first 1.5 years of observations with SPI, Diehl et al. (2005a,b) obtained a spectrum of the $1808.6 \mathrm{keV}$ line from the inner Galaxy of unprecedented quality (see left panel of Fig. 3). The line centroid is unshifted; the line width is intrinsically narrow with a FHWM of about $1.2 \mathrm{keV}$ (and a $2 \sigma$ upper limit of $2.8 \mathrm{keV}$ ), ruling out the earlier GRIS result. These line parameters are consistent with expectations from the Kretschmer et al. (2003) model, which for the inner Galaxy predicts no line shift and a line width of about $1 \mathrm{keV}$ due to Galactic differential rotation (leaving room for a small amount of additional broadening due to modest ISM turbulence around the ${ }^{26} \mathrm{Al}$ sources). The ${ }^{26} \mathrm{Al}$ line is detected with such high significance by SPI that spatially resolved spectroscopy in the inner Galaxy can be performed for the first time, with important implications for the amount and origin of ${ }^{26} \mathrm{Al}$, and the Galactic star formation and SN rates as discussed by Diehl et al. (2005a). 

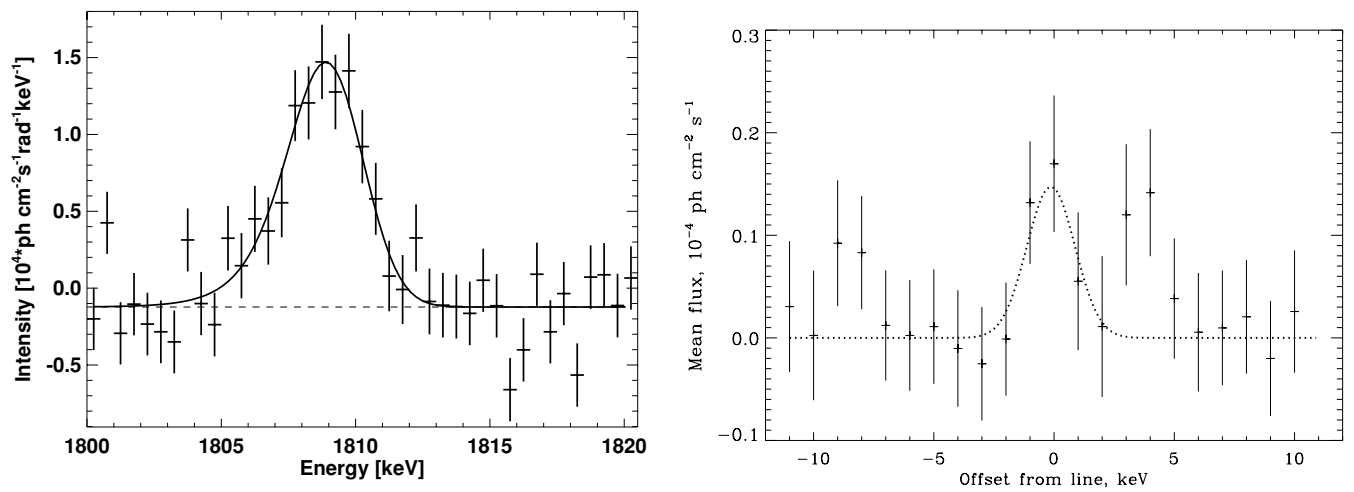

Figure 3. Left panel: the spectrum of the ${ }^{26} \mathrm{Al}$ gamma-ray line emission from the inner Galaxy (data points) and a Gaussian line fit (solid line) as obtained by Diehl et al. (2005b). Right panel: the overlayed and summed spectrum of the observed ${ }^{60} \mathrm{Fe}$ lines at $1173.2 \mathrm{keV}$ and $1332.5 \mathrm{keV}$ (data points) and a Gaussian line fit (dashed line) according to Harris et al. (2005).

\subsection{3. ${ }^{60} \mathrm{Fe}$ and ${ }^{26}$ Al from massive stars}

One of the great successes for SPI was the first significant measurement of ${ }^{60} \mathrm{Fe}$ emission from our Galaxy by Harris et al. (2005) using the observations of the first year. The summed and overlayed spectrum of the two observed lines is depicted in the right panel of Fig. 3. The observed flux per line is $(3.7 \pm 1.1) \times 10^{-5} \mathrm{ph} \mathrm{cm}^{-2} \mathrm{~s}^{-1}$, which translates into a line flux ratio of ${ }^{60} \mathrm{Fe}$ to ${ }^{26} \mathrm{Al}$ of $0.11 \pm 0.03$ per ${ }^{60} \mathrm{Fe}$ line.

The detection of ${ }^{60} \mathrm{Fe}$ has important implications for the nucleosynthesis in massive stars, as was first pointed out by Prantzos (2004, 2005). He integrated the predicted yields for Type II SNe over an initial mass function and found that if he only considered the lower mass range, where the WR phase is absent or weak, the ${ }^{60} \mathrm{Fe}$ to ${ }^{26} \mathrm{Al}$ line flux ratio is significantly overpredicted. This means that there must be an additional ${ }^{26} \mathrm{Al}$ source; Type II SNe do not produce enough ${ }^{26} \mathrm{Al}$. This additional source could be WR stars, because when the integration is extended up to the highest masses, taking into account the ${ }^{26} \mathrm{Al}$ expelled in the WR winds, the observed ${ }^{60} \mathrm{Fe}$ to ${ }^{26} \mathrm{Al}$ line flux ratio can be reproduced. However, one should keep in mind that WR star yields are still uncertain, as they depend sensitively on e.g. metallicity and rotation.

\subsection{Galactic positrons}

\subsubsection{Production processes and positron sources}

The annihilation of positrons with electrons gives rise to two characteristic emissions at gamma-ray energies: the hallmark line at $511 \mathrm{keV}$, and the unique three-photon positronium (Ps) continuum emission (cf. Guessoum, Jean, \& Gillard 2005). The detailed shape of the $511 \mathrm{keV}$ annihilation line, as well as the positronium fraction (the fraction of positrons that annihilate through Ps formation), depend on the physical properties of the annihilation media; therefore detailed spectroscopy of the positron annihilation can provide unique information on the annihilation media and processes.

Positrons can be produced through a variety of processes and by a variety of objects in our Galaxy. The main production processes are $\beta^{+}$decay of radioactive nuclei (formed in SNe, novae, hypernovae, WR stars, and AGB stars), the decay of $\pi^{+}$s resulting from interactions of energetic nuclei (in the ISM), pair (electron-positron) production in $\gamma-\gamma$ interactions (close to compact objects and in gamma-ray bursts), and pair production by electrons in strong magnetic fields (around pulsars). Exotic processes, such as the 


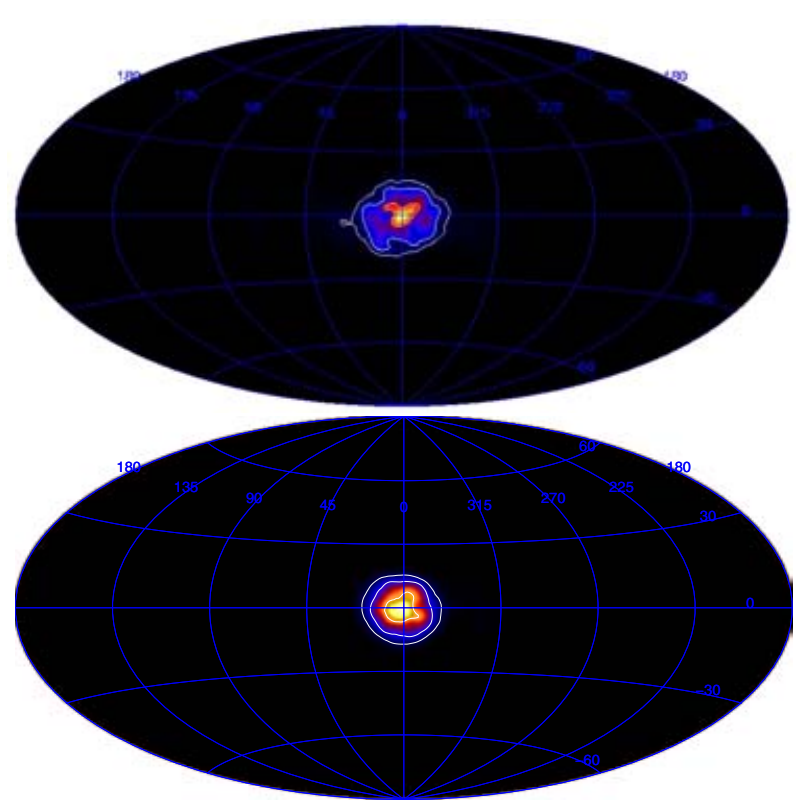

Figure 4. Sky maps in the $511 \mathrm{keV}$ line (top panel, Knödlseder et al. 2005) and the Ps continuum emission (bottom panel, Weidenspointner et al. 2005). The maps were generated employing an implementation of the Richardson-Lucy algorithm. To reduce noise artifacts, the iterative corrections were smoothed during image reconstruction with a $5^{\circ} \times 5^{\circ}$ boxcar average. Further details are given in the text.

annihilation or decay of light dark matter, have also been proposed (Boehm et al. 2004). In spite of this well developed understanding of the fundamental physics of positron production, theoretical models for the positron yield of the various sources are still highly uncertain due to uncertainties concerning the astrophysical conditions in the different sources, and their Galactic distribution and duty cycle. Considering also the limited quality of existing observations, it is not surprising that the origin of the positrons is still poorly understood. At this stage improved measurements of the spatial distribution and the spectrum of the Galactic annihilation radiation are crucial for constraining better the many models.

\subsubsection{Annihilation radiation: Galatic distribution}

Investigations of the sky distribution of the annihilation radiation promise to provide clues to the identification of the source(s) of positrons in our Galaxy. First maps of the annihilation radiation, limited to the inner regions of our Galaxy, were obtained using the OSSE instrument on board the Compton Gamma-Ray Observatory in the $511 \mathrm{keV}$ line and in Ps continuum emission (e.g. Purcel et al. 1997, Milne et al. 2001).

Improved mapping of the $511 \mathrm{keV}$ line is feasible with the commissioning of SPI. During the first year of the mission most of the celestial sphere was observed. Using these observations, first sky maps in the $511 \mathrm{keV}$ positron annihilation line (Knödlseder et al. 2005) and in the Ps continuum emission (Weidenspointner et al. 2005) have been obtained with the SPI spectrometer (Fig. 4, top and bottom). In both maps of positron annihilation radiation, the only prominent signal seen is that from the Galactic bulge region. The surface brightness of any emission from any other sky regions, in particular from the Galactic disk, is much fainter. The emission appears to be symmetric about the Galactic center (GC), and its centroid coincides well with the GC. The differences between the two maps, as well as any apparent small scale sub-structure in the maps, are not significant, as will be demonstrated below.

A more quantitative approach for studying the Galactic distribution of the observed extended emission is model fitting. From modeling the positron annihilation radiation 


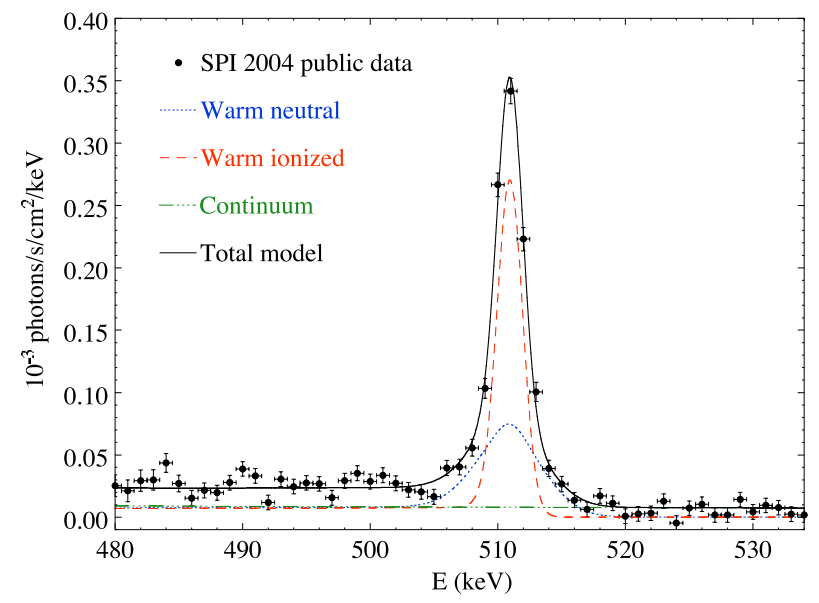

Figure 5. Best fit of the SPI spectrum of the Galactic bulge with the warm components of the ISM and the Galactic continuum emission (from Jean et al. 2005).

by an ellipsoidal distribution with a Gaussian radial profile it can be concluded that the annihilation radiation is spherically symmetric with a FWHM of about $8^{\circ}$ and centered at the GC (Knödlseder et al. 2005, Weidenspointner et al. 2005). The annihilation line signal is strong enough to allow more detailed studies of its Galactic distribution. Knödlseder et al. (2005) fitted a variety of Galactic bulge, halo, and disk models to the data. Stellar bulge and halo models describe the annihilation line emission from the central region of our Galaxy equally well. On adding a Galactic disk component to the model fits, the faint annihilation radiation from the Galactic disk is detected at the 3-4 $\sigma$ level.

From these model fits a total $511 \mathrm{keV}$ line flux from our Galaxy of $(1.5-2.9) \times$ $10^{-3} \mathrm{ph} \mathrm{cm}^{-2} \mathrm{~s}^{-1}$ is derived, with the bulge-to-disk flux ratio being about $1-3$ and the bulge-to-disk annihilation luminosity ratio being even larger with a range of $3-9$. Consequently, the model fits confirm the qualitative mapping result: SPI observations demonstrate for the first time that the annihilation radiation from our Galaxy is dominated by the bulge region (Knödlseder et al. 2005). Assuming a Ps fraction of 0.93 the total $511 \mathrm{keV}$ line flux corresponds to a Galactic positron annihilation rate of $(1.6-2.8) \times 10^{43} \mathrm{~s}^{-1}$.

Another approach to identifying the sources of Galactic positrons is to compare the sky distribution of the $511 \mathrm{keV}$ line emission with all-sky intensity distributions observed at other wavelengths. The relatively best agreement is found at wavelengths dominated by emission from members of old stellar populations, but none of the tracer maps provides an acceptable fit to the data. The sky distribution of positron annihilation appears to be unique, as it is even more bulge dominated than potential old positron source populations such as Type Ia supernovae, novae, or low-mass X-ray binaries (Knödlseder et al. 2005).

Both Knödlseder et al. (2005) and Weidenspointner et al. (2005) searched for evidence for contributions from point sources on top of the extended emission, but none could be found in the $511 \mathrm{keV}$ annihilation line and the Ps continuum emission. Similarly, De Cesare et al. (2004) did not find any evidence for point sources of $511 \mathrm{keV}$ line emission in the GC region using observations by IBIS.

\subsubsection{Annihilation radiation: spectroscopy}

The most detailed spectroscopy to date of the annihilation radiation from the GC region has been performed by Jean et al. (2005). The line is found to be composed of a narrow and a broad component, consistent respectively with the expected line width in a warm ISM for Ps formation by radiative recombination and Ps formation in flight. This result is consistent with an earlier analysis by Churazov et al. (2004). When fitting the annihilation spectra that are expected for the five standard phases of the ISM to the SPI 
spectrum (see Fig. 5), it follows that about half of the positrons annihilate in each of the warm neutral and the warm ionized phases. Possible fitted contributions from annihilations in cold gas, molecular clouds, or hot gas are not significant so far. The importance of annihilations in the warm phases is consistent with our current understanding of positron propagation and annihilation and the gas distribution in the Galactic bulge (Jean et al. 2005). Jean et al. (2005) find a value for the Ps fraction of $0.97 \pm 0.02$, consistent with pre-INTEGRAL results, and with the SPI results obtained by Churazov et al. (2004) and Weidenspointner et al. (2005).

\section{Prospects}

The prospects for further nucleosynthesis studies with INTEGRAL are bright. As the mission continues, we expect to learn much more about the origin of positrons in our Galaxy from ever-improving mapping, spatially resolved spectroscopy, and dedicated observations of individual candidate sources. We also expect to study the Galactic distribution of ${ }^{26} \mathrm{Al}$ in detail by extracting distance information from line shifts, to investigate the Galactic distribution of ${ }^{60} \mathrm{Fe}$ which combined with studies of ${ }^{26} \mathrm{Al}$ will provide new insights into massive star nucleosynthesis as well as the Galactic star formation and SN rates, and to clarify the explosion physics of ccSNe by observing selected SNRs like Cas A.

\section{References}

Boehm, C., et al. 2004, Phys. Rev. Lett. 92, 101301

Churazov, E., et al. 2004, MNRAS 357, 1377

De Cesare, G., et al. 2004, Advances in Space Research, in press

Diehl, R., Prantzos, N. \& von Ballmoos, P. 2005, Nucl. Phys. A, in press

Diehl, R., et al. 2005a, Nature, submitted

Diehl, R., et al. 2005b, A\&\&A, submitted

Guessoum, N., Jean, P. \& Gillard, W. 2005, A\&A 436, 171

Harris, M.J., et al. 2005, A\&\&A 433, L49

Jean, P., et al. 2005, A\& $A$ submitted

Knödlseder, J., et al. 1999, A\&A 344, 68

Knödlseder, J., et al. 2005, $A \& A$, in press

Kretschmar, P. 2005, these proceedings

Kretschmer, K., Diehl, R. \& Hartmann, D.H. 2003, A\&\&A 412, L47

Leising, M.D., \& Share, G.H. 1994, ApJ 424, 200

Milne, P.A., et al. 2001, Proc. of Gamma 2001 (AIP 587), 11

Naya, J.E., et al. 1996, Nature 384, 44

Prantzos, N. 2004, A\&̈A 420, 1033

Prantzos, N. 2005, Proc. $5^{\text {th }}$ INTEGRAL Science Workshop ESA SP-552, 15

Purcell, W.R., et al. 1997, ApJ 491, 725

Renaud, M., et al. 2004, Proc. $5^{\text {th }}$ INTEGRAL Science Workshop ESA SP-552, 81

Sturner, S.J., \& Naya, J.E. 1999, ApJ 526, 200

Weidenspointner, G., et al. 2005, A\&A, submitted

Woosley, S.E. \& Weaver, T.A. 1994, ApJ 423, 371 


\section{Discussion}

GARCIA: Is 511 a point source?

Weidenspointner: No: It is extended - must be at least 3 point sources or more.

VÖLK: Regarding ${ }^{44} \mathrm{Ti}$ sources you said that only Cas A has been detected by INTEGRAL. Concerning the consistent claims of the past, concerning ${ }^{44} \mathrm{Ti}$ emission from the SNR Vela Jr., what is then your position?

WeIDENSPOINTNER: First INTEGRAL upper limits based on observations taken during Cycle 1 are still less constraining than previous COMPTEL results. By the end of Cycle 3 the exposure to Vela Jr. will have increased by more than a factor of 3 though, and then INTEGRAL should be able to provide a better answer to this intriguing question.

Bosch-RAmon: Is the line e-e+ of the great annihilator completely ruled out?

Weidenspointner: If the Great Annihilator is manifesting itself as a point source, or point-like source, of narrow $511 \mathrm{keV}$ line emission, then it can indeed be completely ruled out.

ELVIS: 1 . The ps $+511 \mathrm{keV}$ maps appeared to show structure. Are these significant? 2. The ${ }^{60} \mathrm{Fe}$ line had similar sized features at slightly higher and lower energies, which suggests that ${ }^{60} \mathrm{Fe}$ may not be a significant feature. If treated as an upper limit does this solve the $\mathrm{Fe} / \mathrm{Al}$ ratio problem?

Weidenspointner: 1 . No. We have not yet found any significant structure on angular scales of a few degrees or below.

2. One should keep in mind that the combined significance of the two high-energy ${ }^{60} \mathrm{Fe}$ lines is only $3.4 \sigma$. The feature above the overlayed ${ }^{60} \mathrm{Fe}$ lines is the residual of a well known background line from ${ }^{69} \mathrm{Ge}$ decay in the detectors, which our analysis reduces by about a factor of 100 . The weak residual below the ${ }^{60} \mathrm{Fe}$ lines cannot be attributed to a single instrumental background feature. If the detected flux is treated as an upper limit, the need for a source of ${ }^{26} \mathrm{Al}$ in addition to ccSNe is even exacerbated. 